\title{
Inhibition and Cognitive Load in Fractions and Decimals
}

\author{
Vana A. Avgerinou*1 and Andrew Tolmie ${ }^{2}$ \\ ${ }^{1}$ Putney High School (Girls' Day School Trust) \\ ${ }^{2}$ UCL Institute of Education, University College London, UK
}

*Corresponding author information: Vana A. Avgerinou, Putney High School (GDST) 35 Putney Hill London SW15 6BH, London, UK (e-mail: v.avgerinou@put.gdst.net).

Length: 4999 words, excluding abstract, references, tables and figures.

\section{Acknowledgements:}

The authors would like to thank the teachers and children from participating schools. Special thanks are due to the Unlocke team at Birkbeck University for providing the Whack-A-Mole task, to Hannah Wilkinson for her valuable feedback at the early stages of the project, to Su Morris for sharing the code for the Stroop task and to Katie Gilligan for her help on the use of the Open Sesame software. The first author gratefully acknowledges the support of the research community in Putney High School. 


\title{
Inhibition and Cognitive Load in Fractions and Decimals
}

\author{
Abstract: \\ Background. Prior research with adults and children suggests that inhibitory control may have a role \\ to play in learning counterintuitive fractions and decimals that are inconsistent with whole number \\ knowledge. However, there is little research to date with primary-school aged children at the early \\ stages of fraction and decimal instruction that addresses this relationship. Understanding this \\ association has the potential to inform instructional practices concerning the learning of \\ counterintuitive maths concepts.
}

Aims. This study examined the relationship between inhibitory control and counterintuitive fractions and decimals in the presence of varying cognitive load in 8-10 year-old children.

Method. Children aged 8 to 10 years $(N=95)$ completed a fraction and decimal magnitude comparison task with pairs that were either consistent (controls) or inconsistent (counterintuitive) with whole number magnitudes. Cognitive load was manipulated by presenting trials with simple integrated text (no additional load), with integrated text accompanied by supportive illustrations (low load) or with illustrations containing information that needed to be integrated to arrive at an answer (high load). Participants also completed measures of response and semantic inhibition.

Results. Inhibitory control uniquely contributed to performance in counterintuitive fractions and decimals only under conditions of high cognitive load, where low semantic inhibition predicted longer response times.

Conclusion. Our results indicate a more nuanced relation between inhibitory control and counterintuitive fractions and decimals than presumed by previous research. They suggest that the role of inhibitory control when reasoning about counterintuitive fractions and decimals is not constant, and it is only drawn on at high levels of cognitive load. 
Keywords: Inhibitory control, cognitive load, counterintuitive learning, primary maths, magnitude comparison, fractions, decimals, illustrations. 
Research has highlighted the role of inhibition in learning counterintuitive concepts in mathematics and science (Mareschal, 2016; Vosniadou, 2014), but no study has investigated if performance on semantic and response inhibition measures predicts counterintuitive reasoning in the specific context of fractions and decimals. Inhibitory control is not a constant, however- as part of the executive function system, the level of ability necessary to apply it varies as function of cognitive load (Basanovic, Notebaert, Clarke, MacLeod, Jawinski, \& Chen, 2018; Berggren, Richards, Taylor, \& Derakshan, 2013). In maths learning, one common reason for variation in cognitive load is the use and layout of illustrations accompanying problems. The twin goals of this study are therefore to examine the impact of inhibitory control on reasoning about fractions and decimals, and to do so under realistic conditions of varying cognitive load.

\section{Counterintuitive Reasoning in Fractions and Decimals}

Thinking in whole numbers serves children well during the early school years, but may hinder their understanding of rational numbers (Vosniadou, 2014). This is particularly true in the case of fractions and decimals, where the two whole numbers separated either by a line, in a fraction, or a point, in a decimal, prompt a child to treat them as separate quantities rather than a unified whole (Roell, Viarouge, Houde, \& Borst, 2017; Vosniadou, 2014; Vosniadou, loannides, Dimitrakopoulou, \& Papademetriou, 2001; Vosniadou \& Verschaffel, 2004). For example, children commonly reason that $2 / 7$ is greater than $2 / 5$ because 7 is greater than 5 , or that 1.25 is greater than 1.3 because 25 is greater than 3.

Children's attempts to assimilate these apparent inconsistencies in their understanding of numbers lead to systematic errors in performance (Resnick, Nesher, Leonard, Magone, Omanson, \& Peled, 1989; Vamvakoussi \& Vosniadou, 2010; Vosniadou \& Verschaffel, 2004). These errors, known as 'whole number bias' (Ni \& Zhou, 2005), are resistant to change. They may remain even after the new 
concept has been securely understood (Dunbar, Fugelsang, \& Stein, 2007; Mareschal, 2016; Masson, Potvin, Riopel, \& Foisy, 2014; McNeil \& Alibali, 2005), and have been documented not only in school children and adults (Durkin \& Rittle-Johnson, 2015; Lai \& Wong, 2017; Meert, Gregoire, \& Noel, 2010; Stafylidou \& Vosniadou, 2004; Vamvakoussi \& Vosniadou, 2010) but also in skilled mathematicians (Obersteiner, Van Dooren, Van Hoof, \& Verschaffel, 2013). In contrast, fractions and decimals that are consistent with the way children have learnt to reason about whole numbers (e.g. 4/5 >3/5 because 4 > 3) do not elicit similar errors (Nunes \& Bryant, 2008; Stafylidou \& Vosniadou, 2004). The ability to manage these counterintuitive concepts and perform proficiently in operations involving rational numbers predicts later achievement in mathematics (Bailey, Hoard, Nugent, \& Geary, 2012; Siegler, Duncan, Davis-Kean, Duckworth, Claessens, Engel et al., 2012) and occupational success (LortieForgues, Tian, \& Siegler, 2015).

\section{The role of inhibitory control in fraction and decimal reasoning}

Inhibitory control (IC), the ability to stop or override a dominant mental or motor response (MacLeod, 2007), belongs to a set of core skills known as executive functions (EF) (Miyake, Friedman, Emerson, Witzki, Howerter, \& Wager, 2000). Response inhibition is the suppression of a dominant motor response that has been previously reinforced, whereas semantic inhibition is the stopping of an automatic mental response when faced with a stimulus that elicits competing reactions (Nigg, 2000). There is evidence that EF correlate with mathematical achievement in school-age children and pre-schoolers (Bull \& Scerif, 2001; Espy, McDiarmid, Cwik, Stalets, Hamby, \& Senn, 2004), and it has been suggested that IC may be specifically involved in accurate performance with fractions and decimals (Gómez, Jiménez, Bobadilla, Reyes, \& Dartnell, 2015; Lai \& Wong, 2017; Meert et al., 2010; Roell et al., 2017; Vosniadou, 2014). 
Meert et al. (2010) reported that 10-12 year-olds were faster at comparing fractions with common denominators (e.g. $2 / 7 \vee 5 / 7$ ) in which the numerators were consistent with whole number ordering $(2<5)$ compared to fractions with different denominators (e.g. $2 / 3 \vee 2 / 5)$ where the greater fraction (2/3) was made up of the smaller denominator (3). Roell et al. (2017) found that French $7^{\text {th }}$ Graders (average age 12.5 years) took longer to compare two decimal numbers (e.g. $1.25 \vee 1.3$ ) in which the smaller number had the greatest number of digits after the decimal point compared to decimal numbers that were consistent with the whole number principle that numbers with more digits are larger (e.g. 2.15 v 2.1). Research with adults point to similar findings (M. DeWolf, Grounds, Bassok, \& Holyoak, 2014; Vamvakoussi, Van Dooren, \& Verschaffel, 2012). Although not all studies report this pattern (Ninaus, Kiili, McMullen, \& Moeller, 2017), it has been proposed that the whole number bias happens because the brain automatically processes whole numbers, and consequently the digits that make up a fraction or decimal (Kallai \& Tzelgov, 2011). Vamvakoussi et al. (2012) and Melissa DeWolf and Vosniadou (2011) suggest that comparing counterintuitive fraction and decimal pairs takes longer and is less accurate than comparing intuitive pairs because the former involve significant IC demands to suppress interference from prior number knowledge and apply correct but counterintuitive principles. Those with better IC should therefore exhibit less pronounced effects.

The only study to our knowledge that has investigated this possibility, with Chilean 5th to 7th graders (ages 10-12) using fractions (Gómez et al., 2015), found no unique role of semantic inhibition in counterintuitive fraction reasoning after controlling for general math achievement. However, it is possible that the children who took part in this study might have had sufficient practice to have automated inhibition of the processing biases involved in reasoning about counterintuitive fractions. A repetition with younger children at the early stages of learning about the counterintuitive operations involved in both fractions and decimals is therefore needed to establish whether or not there is evidence of an effect. 


\section{The role of Cognitive Load (CL)}

The use and layout of illustrations in mathematical problems has been linked to increased $\mathrm{CL}$ and poorer performance (Berends \& Lieshout, 2009; Elia, Gagatsis, \& Demetriou, 2007). One explanation for this, the redundancy effect, suggests that processing illustrations that are irrelevant or unnecessary increases CL because it wastes limited cognitive resources (Mayer, Heiser, \& Lonn, 2001). $\mathrm{CL}$ may also be increased by high element interactivity, when a learner is required to process and integrate physically separated sources of information (e.g. text and illustration) (Ayres \& Sweller, 2005). These pieces of information must be first processed in isolation and subsequently integrated, a process that is cognitively demanding and slows down processing (Sweller \& Chandler, 1994).

Applying this to counterintuitive fraction or decimal comparisons, we expected working memory to already be taxed by the cognitive effort required to solve the task. We therefore anticipated that where illustrations are used as vehicle to display information that must be integrated, there will be fewer resources available to inhibit prior number knowledge. This would lead to slower processing and lower accuracy, perhaps especially among those with lower levels of IC, compared to items consisting of integrated text with supporting illustration or integrated text only. To test this, we created three sets of problems of varying CL. In the first set, termed 'high load' (HL), illustrations are used as a vehicle to naturally decompose elements of numerical information which then must be integrated in order to reach an answer. This set was designed to induce high element interactivity. In the second set, termed 'low load' (LL), information is presented in integrated text statements with a supportive illustration which does not itself need to be processed to arrive at an answer. This set was designed to induce redundancy effects. In the third set, termed 'no additional load' (NAL) information is presented in simple integrated text without illustration, to provide a baseline measure. 


\section{Current Study}

This study therefore aims to examine the influence of inhibitory control on processing counterintuitive fractions/decimals in children aged 8 to 10 , under conditions of varying cognitive load. We test the following hypotheses:

1. Responses will be slower and less accurate in trials where the fraction/decimal magnitude comparisons are inconsistent with whole number magnitude (counterintuitive) compared to trials where these are consistent (control).

2. Responses will be slower and less accurate in trials where illustrations are used to create effects of redundancy $(\mathrm{LL})$ and high element interactivity $(\mathrm{HL})$, with these effects being more pronounced for HL than LL trials.

3. There will be an association between IC measures and performance in the counterintuitive trials after controlling for age and performance in control trials, those with better IC exhibiting better performance. We expected this association to be stronger where $\mathrm{CL}$ is greater because fewer resources will be available to activate IC.

\section{Method}

\section{Design}

A combined experimental/correlational design was employed, focused on a fraction/decimal magnitude comparison task with comparison pairs that were either consistent (control) or inconsistent with whole number magnitudes (counterintuitive). Items of both types were further subdivided into pairs that presented information in simple integrated text (NAL condition), pairs with a supportive illustration that did not need processing to arrive at an answer (LL condition), and pairs that used illustrations as a vehicle to naturally decompose elements of information that needed to be integrated in order to reach an answer (HL condition). Semantic inhibition was measured using a non- 
numerical version of the Stroop task. Response inhibition was measured using the Whack-A-Mole version of the Go/No Go task.

\section{Participants}

A total of 95 school children in Year 4 (Y4) (N=38, 33 girls) and Year 5 (Y5) (N=57, 38 girls) from a selective private school for girls and a mixed state school in high socio-economic areas of London took part in this study. These year groups correspond approximately to children aged 8-9 and 9-10 (overall mean age $=9.7$ years, $S D=0.6)$. Written parental permission and child assent was obtained for all participants. Procedures were approved by the authors' institutional research ethics committee.

\section{Measures}

\section{Fraction/decimal magnitude comparison task}

This task was designed using Open Sesame 3 software. It consisted of 24 fraction pairs (12 control and 12 counterintuitive) and 24 decimal pairs (10 control and 14 counterintuitive; due to an initial classification error, two decimal counterintuitive items were labelled as controls, one NAL and one LL). On each trial, participants read a statement on-screen about which of two numbers was smaller or larger and pressed one of two keys to indicate whether they thought this was correct/incorrect. For fractions, control trials consisted of pairs with common denominators but different numerators, counterintuitive of pairs with common numerators where the greater denominator belonged to the smaller fraction (e.g. $3 / 9<3 / 4$ ). For decimals, control pairs consisted of numbers in which the larger one had more digits and greater magnitude after the decimal point (e.g. $7.59 \vee 7.3)$, counterintuitive of numbers where this was reversed (e.g. $8.39 \vee 8.6)$. Control and counterintuitive trials across fraction/decimal pairs were matched in terms of statement length, positive versus negative wording, numerical distances of the whole numbers, and for decimals, the number of digits after the point. There were two types of statement: those in which the intuitive response is to say that the statement 
is correct, but for counterintuitive items it is really incorrect; and vice versa. There were also four types of response, balanced across control and counterintuitive trials: greater false, greater true, smaller false, smaller true.

Illustrations consisted of a set of clip art characters designed for classroom lessons (www.mycutegraphics.com). The condition of CL varied systematically, so that fraction and decimal pairs were presented without illustrations ( $N A L, N=16$ ), with supportive illustrations ( $L L, N=16$ ) or with illustrations in which relevant information needed integrating $(H L, N=16)$ (Figures $1 a, 1 b, 1 c)$. The task started with four practice trials (no criterion for progression was set), followed by two trial blocks separated by a self-timed break. For half the participants in each age group, Block 1 consisted of all the fraction pairs (Fractions First) and block 2 of all the decimal pairs, each presenting 24 trials in randomised sequence; the other half completed the blocks in the reverse order (Decimals First). Each trial was preceded by a fixation cross of 500 milliseconds (ms) duration and stimuli stayed on the screen until a keypress response $(\mathrm{x}=$ correct, $\mathrm{m}=$ incorrect $)$ was made. Feedback was not provided. Accuracy and response times (RT) were recorded and averages were computed for each trial type.

\section{Semantic inhibition: Animal Size Stroop Task}

In order to avoid confounding with numerical ability, an animal-size Stroop task based on Merkley, Thompson, and Scerif (2015) was used to assess participants' ability to ignore pre-potent irrelevant information. Participants saw a large $(105 \times 70 \mathrm{~mm})$ and a small $(45 \times 30 \mathrm{~mm})$ animal image on the computer screen and had to press a key to indicate which animal was larger in real life. On congruent trials the largest animal in real life was also the larger image on the screen. On incongruent trials the largest animal in real life was the smaller image on the screen. The task consisted of two experimental blocks, each containing 36 pseudo-randomised trials with an equal number of left and right keypress responses and congruent and incongruent trials. The stimuli stayed on the screen for a maximum of 
3000 milliseconds and were separated by an inter-trial gap of 500 milliseconds. Differences in mean accuracy (accuracy cost) and response times (RT cost) between congruent and incongruent trials were used as measures of IC performance. Higher values indicate lower levels of semantic inhibition.

\section{Response inhibition: Whack-A-Mole (W-A-M) task}

The W-A-M task, based on Casey, Trainor, Orendi, Schubert, Nystrom, Giedd et al. (1997) was used to assess participants' ability to withhold a dominant motor response. Children were instructed to press the space bar on the keyboard as quickly as possible when a cartoon mole appeared on the screen (Go trial), but not to press it when a cartoon vegetable appeared (No Go trial). The task consisted of 75 Go trials and 25 No Go trials, which were randomised and distributed into two blocks. The stimuli stayed on the screen for 1.8 seconds. Mean RT for Go trials and mean accuracy for Go and No Go trials were used as measures.

\section{Procedure}

Participants were tested individually in a quiet room during school hours. They were asked to read the instructions on a $15.6 \mathrm{in.} \mathrm{laptop} \mathrm{screen} \mathrm{before} \mathrm{starting} \mathrm{each} \mathrm{task.} \mathrm{The} \mathrm{fraction/decimal} \mathrm{magnitude} \mathrm{task}$ was performed first, followed by the Stroop task, and then the W-A-M task. The sessions took on average 20 minutes.

\section{Results}

The first set of analyses considers data from the magnitude task. It examines if responses differ across number format and analyses the data for fractions and decimals separately using a Repeated Measures (RM) ANOVA. The second reports the results from the IC measures. The third assesses the results from multiple regressions examining predictors of magnitude task performance. 
In line with convention, mean RTs are reported for correct trials in the Stroop task and Go trials in the W-A-M task; and for all trials in the magnitude comparison task. Participants whose mean accuracy or RT was further than \pm 3.29 standard deviations away from the overall group mean in the specific tasks were excluded from the analysis (Field, 2013), leaving a total of 90 participants for the RM analyses and 89 for the regressions (because of exclusions in the W-A-M task).

\section{Magnitude Task}

Accuracy and RT scores (Table 1) indicated sufficient differences between fraction and decimal performance to necessitate analysing them separately. These differences were explored with a twoway RM ANOVA on accuracy and RT with trial type (2 levels: counterintuitive, control) and number format (2 levels: fractions, decimals) as within-subjects factors. There was a main effect of congruency on accuracy, $F(1,89)=64.59, p<.001$, partial $\eta p^{2}=.42$, and RT, $F(1,89)=53.06, p<.001$, partial $\eta_{p}^{2}=$ .37 , with more accurate and faster responses to control trials compared to counterintuitive. There was also a main effect of number format on accuracy, $F(1,89)=7.43, p=.008$, partial $\eta p^{2}=.08$, and an interaction, $F(1,89)=15.16, p<.001$, partial $\eta_{p}{ }^{2}=.14$, which was due to counterintuitive trials, but not control, attracting significantly more accurate responses in fractions than decimals. There was also an interaction on RTs, $F(1,89)=42.36, p<.001$, partial $\eta_{p}{ }^{2}=.32$, which was due to performance in counterintuitive trials, but not control, being slower in fractions compared to decimals. There was no main effect of number format on RT.

\section{Fractions}

Alpha for accuracy on control fractions was .616 and for counterintuitive was .680. A two-way RM ANOVA with trial type (2 levels: counterintuitive, control) and $C L$ (3 levels: NAL, LL, HL) as within- 
subjects factors was performed on accuracy and RTs separately. Mauchly's test indicated that the assumption of sphericity for the main effect of illustration had been violated, therefore GreenhouseGeiser corrected tests are reported. Pairwise comparisons for main effects of illustration are corrected using a Bonferroni adjustment, corrected $p=.05 / 3=.017$

There was a significant main effect of trial type, with higher accuracy $F(1,89)=14.8, p<.001$, partial $\eta_{\mathrm{p}}{ }^{2}=.14$, and shorter RTs, $F(1,89)=69.99, p<.001$, partial $\eta_{\mathrm{p}}^{2}=.44$ for control compared to counterintuitive trials (Figures 2 and 3). There was a main effect of CL on RTs, with the fastest RTs in NAL, followed by LL, followed by HL trials, $F(1.87,166.41)=50.54, p<.001$, partial $\eta_{p}{ }^{2}=.36$. Pairwise differences were significant between $\mathrm{HL}$ and $\mathrm{LL}$ trials and between the $\mathrm{HL}$ and NAL trials only.. There was a significant main effect of $C L$ on accuracy, $F(1.80,160.26)=5.21, p=.008$, partial $\eta_{p}^{2}=.05$, revealing more accurate responses in $\mathrm{HL}(M=.88, S E=.015)$ compared to $\mathrm{LL}$ trials $(M=.83, S E=.019)$ across both control and counterintuitive fractions. There was no significant difference between $L L$ and NAL and no interaction.

\section{Decimals}

Alpha for accuracy on control trials was .445 (due in part to the smaller number of items, and generally weak inter-item correlations, for two items in particular) and for counterintuitive was .92. There was a significant main effect of trial type with higher accuracy, $F(1,89)=49.57, p<.001$, partial $\eta_{p}^{2}=.36$ and shorter RTs, $F(1,89)=8.25, p=.005$, partial $\eta_{p}{ }^{2}=.09$ for control compared to counterintuitive trials (Figures 4 and 5). There was also a main effect of $C L$ on RTs, $F(1.95,173.91)=$ 35.81, $p<.001$, partial $\eta_{p}{ }^{2}=.29$, with highest RTs for HL trials, followed by LL, followed by NAL trials. All pairwise differences were significant. 
In summary, in the magnitude task, children took longer and were less accurate in comparing the magnitude of both fraction and decimal pairs in counterintuitive compared to control trials. As expected, children were also significantly faster in magnitude comparison in the NAL trials compared to $\mathrm{LL}$ and $\mathrm{HL}$ trials across both fraction and decimal pairs. Contrary to expectations, performance in $\mathrm{HL}$ fraction pairs were more accurate than LL pairs (the differences in decimals were non-significant). There was no evidence that $C L$ interacted with the increased demands of counterintuitive items in either the fraction or the decimal trials.

\section{Inhibitory control tasks}

The results of the inhibitory control tasks are summarised in Table 2 . Children responded faster, $t$ (89) $=-9.21, p<.001, d=0.9$, and more accurately, $t(89)=5.71, p<.001, d=0.6$, in congruent compared to incongruent trials in the Stroop task. Children were also significantly more accurate in Go trials compared to no-Go trials, $t(88)=8.08, p<.001, d=0.86$ in the W-A-M task.

\section{Regression Analyses}

Cronbach's alpha values were examined for counterintuitive fractions and decimals within each level of $\mathrm{CL}$. Although there was a slight dip for HL accuracy, the remainder indicated sufficiently good internal consistency at this level to justify combined analysis. Respective values for accuracy and RT were .737 and .690 for NAL trials, .702 and .775 for LL trials and .581 and .746 for HL trials. Regressions were run on accuracy and RT separately to examine if IC could account for variance in the counterintuitive trials within each level of $\mathrm{CL}$ after controlling for age and performance in the control trials. In block 1, the regression models included age and mean accuracy or RT in the control trials, as baseline performance was expected to have an influence on the outcome variable. The Stroop variables were entered in block 2: accuracy cost (congruent minus incongruent trials) and RT cost 
(incongruent minus congruent trials). The W-A-M variables were entered in block 3: Go accuracy, No Go accuracy and Go RT.

Regression on counterintuitive accuracy showed that neither semantic nor response inhibition was significantly associated with counterintuitive accuracy after controlling for age and accuracy in control trials. This pattern was the same across all levels of $\mathrm{CL}$, with only Go accuracy being significantly positively associated with counterintuitive accuracy. The regression models for counterintuitive RT (Table 3) were more revealing, and explained variance was consistently higher than for accuracy suggesting that accuracy is a more noisy measure, affected by a number of factors beyond those considered here. Regression on NAL RT indicated that only performance in NAL control trials predicted performance in counterintuitive NAL trials; the lack of age effect here may signal that age had an impact on children's familiarity with and accuracy on both types of problem, but not on their basic speed of responding. Regression on LL RT indicated that Go accuracy was significantly associated with LL counterintuitive RT. This was a negative association, such that higher Go accuracy predicted shorter RTs, indicating that children who scored higher in general processing accuracy responded faster to the LL counterintuitive trials. Regression on HL counterintuitive RT revealed that in line with Hypothesis 3, Stroop accuracy cost was a significant predictor of counterintuitive RT, uniquely accounting for $3.4 \%$ of variance after controlling for age and performance in $\mathrm{HL}$ control trials. The final beta for this association had a probability of .019, significant at conventional levels and still marginally so with a Bonferroni correction for comparison across three levels of $\mathrm{CL}$. The relationship was positive, such that higher accuracy cost (worse semantic IC) was associated with longer RTs.

In summary, the regression analyses revealed a unique role for semantic inhibition only when cognitive load was increased during the HL trials and only for RTs. Go accuracy predicted counterintuitive accuracy for the NAL and LL items and counterintuitive RT for the LL items. There was no sign that IC was involved in these trials. But for HL items, semantic inhibition, as assessed by 
accuracy cost, was predictive of RT alongside Go accuracy - the higher the cost (the worse the IC) the slower the responses. This finding suggests that in the presence of increased cognitive load, children with lower scores in semantic inhibition were slower to respond. In contrast, performance appeared to be sufficiently automated in the NAL and LL items that general processing ability (as measured by Go accuracy) was enough to predict performance.

\section{Discussion}

We tested three hypotheses with regard to performance on counterintuitive fractions and decimals in the presence of varying cognitive load in 8 to 10 year old children. Results relating to each are considered in turn below.

\section{The role of whole number bias in counterintuitive fractions and decimals}

In line with previous work (Melissa DeWolf \& Vosniadou, 2015; Gómez et al., 2015; Stafylidou \& Vosniadou, 2004), the first hypothesis proposed that children's responses would be slower and less accurate on counterintuitive compared to control trials. The data for both fractions and decimals supported this hypothesis, suggesting that children's performance was influenced by intuitive responses (i.e. consistent with whole number magnitudes) and that it takes longer to reach the correct answer, with less certain outcome, in trials that go against operations of whole numbers. These findings add to the increasing body of evidence proposing that the hard-won knowledge of whole numbers that has helped children secure basic number facts in the first years of schooling adversely interferes with their efforts to construct a competent understanding of fraction and decimal concepts.

\section{The role of cognitive load in counterintuitive fractions and decimals}

The second hypothesis predicted that responses would be slower and less accurate in $\mathrm{HL}$ compared to LL and NAL trials. It was anticipated that HL trials would leave fewer resources available to address the 
cognitive effort required to control interference from whole number bias. As expected, performance in $\mathrm{HL}$ items across fractions and decimals was slowest, followed by LL, followed by NAL. However, children were significantly more accurate in HL fractions across both control and counterintuitive trials, and for decimals there was no effect. The slower RTs for HL fractions indicate that accuracy levels were bought at the expense of RTs. This finding suggests that when faced with problems that induced greater cognitive load children tended to slow down to ensure accuracy, indicating that they were aware of the increase in load, and adjusted performance accordingly. This may also have been a factor in the increased RTs for counterintuitive trials.

\section{The role of inhibitory control in the presence of varying cognitive load}

The third hypothesis proposed that there would be an association between IC scores and performance in counterintuitive trials and that this association would be stronger for $\mathrm{HL}$ trials because there would be fewer resources available to activate IC. Although children's prior knowledge of whole number magnitudes interfered with their reasoning in counterintuitive trials across all levels of $C L$, making their performance slower and less accurate, there was no evidence that the IC measures predicted performance in the NAL and LL counterintuitive trials after controlling for age and performance in the respective control trials. Only accuracy in Go trials was consistently associated with performance in these trials, such that higher Go accuracy predicted more accurate and faster responses. Since the effects of Go accuracy were in addition to the effects of performance on the corresponding control trials, and any influence general processing ability may have had on those, the implication is that the counterintuitive trials drew on something extra. One possibility is that this measure reflects confidence in responding accurately, and this has greater impact on the more difficult counterintuitive trials.

However, when $\mathrm{CL}$ increased due to the demands placed by the need to integrate numerical information, IC contributed unique variance to children's RT performance, alongside Go accuracy. 
Under high CL better semantic inhibition predicted faster responses, and conversely, poorer inhibition led children to slow down further. This novel finding suggests a more nuanced relation between IC and counterintuitive fractions and decimals than presumed by recent research. In particular, it indicates that the role of inhibitory control when reasoning about counterintuitive fractions and decimals is not constant, and it is only drawn on at high levels of cognitive load, instigating increased care among those for whom the demands are more challenging. This is again consistent with the idea that children - in this sample at least - were capable of adjusting their performance when they perceived task demands to be higher. At lower levels of demand, this adjustment appears to be automatic, but at high levels to be more deliberate.

\section{Limitations}

The participants in this study came from areas with high socioeconomic status (SES), with the majority $(\mathrm{N}=67)$ attending a selective private school. Previous studies have demonstrated that IC is affected by variations in SES, with children from high SES backgrounds performing better on IC tasks than their low-SES peers (Hackman, Gallop, Evans, \& Farah, 2015; Noble, McCandliss, \& Farah, 2007; Spielberg, Galarce, Ladouceur, McMakin, Olino, Forbes et al., 2015). The data for the IC tasks showed a reasonable spread of scores (as indicated by their standard deviations), however, so the lack of association between IC measures and performance in the NAL and LL counterintuitive trials cannot be explained on the basis of low (and therefore unpredictive) variance. The alternative possibility, that the children who took part in this study had already progressed to the point where IC was well established for problems of this type, and it was effectively automated, is more plausible. It is unclear if the impact of inhibitory control on counterintuitive fractions and decimals would be limited to trials associated only with high CL in a low-/mixed-SES sample. Future research should investigate this.

Semantic inhibition was measured using a non-numerical Stroop task, in order to avoid numerical ability becoming a confounding factor. However, given that children needed to ignore the whole 
numbers in fractions and decimals in order to process them appropriately, it is possible that different outcomes would have been obtained if semantic inhibition had been measured using a numerical Stroop task in which participants were asked to report the physical size of the number and ignore its magnitude (Bugden \& Ansari, 2011). Previous research (Gilmore, Keeble, Richardson, \& Cragg, 2015) found that IC was related to arithmetic performance only on a numerical, but not a non-numerical task (Animal Stroop).

\section{Conclusion}

In order to understand how children learn new concepts in mathematics it is important to grasp the role of processing biases where these go against established understandings of number magnitudes. This study contributed to this by exploring the influence of whole number bias and the role of IC and $\mathrm{CL}$ in counterintuitive fractions and decimals. To our knowledge, this is the first attempt directly to examine the association between response and semantic inhibition and counterintuitive fractions and decimals in children who are at the early stage of fraction and decimal instruction. The data are in line with previous findings that fractions and decimals that go against conceptions of whole number magnitude are associated with poorer performance. The present work revealed a more complicated relationship between inhibition and performance than suggested by previous research, indicating that the role of IC varies as a function of $\mathrm{CL}$, and that children are capable of managing load deliberately by slowing down where necessary, and even of automating such adjustments, so that individual variation

in tasks measuring IC skills is not predictive. Future research looking at the role of IC in counterintuitive learning must develop a sufficiently nuanced approach to allow for these kinds of complex effects. 


\section{References}

Ayres, Paul, \& Sweller, John. (2005). The split-attention principle in multimedia learning. In The cambridge handbook of multimedia learning. (pp. 135-146). New York, NY, US: Cambridge University Press.

Bailey, Drew H., Hoard, Mary K., Nugent, Lara, \& Geary, David C. (2012). Competence with fractions predicts gains in mathematics achievement. Journal of Experimental Child Psychology, 113(3), 447-455. doi:10.1016/j.jecp.2012.06.004

Basanovic, Julian, Notebaert, Lies, Clarke, Patrick, MacLeod, Colin, Jawinski, Philippe, \& Chen, Nigel. (2018). Inhibitory attentional control in anxiety: Manipulating cognitive load in an antisaccade task (Vol. 13).

Berends, Inez E., \& Lieshout, Ernest C. D. M. Van. (2009). The effect of illustrations in arithmetic problem-solving: Effects of increased cognitive load. Learning and Instruction, 19(4), 345353. doi:10.1016/j.learninstruc.2008.06.012

Berggren, Nick, Richards, Anne, Taylor, Joseph, \& Derakshan, Nazanin. (2013). Affective attention under cognitive load: Reduced emotional biases but emergent anxiety-related costs to inhibitory control. Frontiers in human neuroscience, 7, 188-188. doi:10.3389/fnhum.2013.00188

Bugden, S., \& Ansari, D. (2011). Individual differences in children's mathematical competence are related to the intentional but not automatic processing of arabic numerals. Cognition, 118(1), 32-44. doi:10.1016/j.cognition.2010.09.005

Bull, Rebecca, \& Scerif, Gaia. (2001). Executive functioning as a predictor of children's mathematics ability: Inhibition, switching, and working memory. Developmental Neuropsychology, 19(3), 273-293. doi:10.1207/S15326942DN1903_3

Casey, B. J., Trainor, R. J., Orendi, J. L., Schubert, A. B., Nystrom, L. E., Giedd, J. N., Castellanos, F. X., Haxby, J. V., Noll, D. C., Cohen, J. D., Forman, S. D., Dahl, R. E., \& Rapoport, J. L. (1997). A developmental functional MRI study of prefrontal activation during performance of a go-nogo task. J Cogn Neurosci, 9(6), 835-847. doi:10.1162/jocn.1997.9.6.835

DeWolf, M., Grounds, M. A., Bassok, M., \& Holyoak, K. J. (2014). Magnitude comparison with different types of rational numbers. Journal of Experimental Psychology: Human Perception and Performance, 4O(1), 71-82. doi:10.1037/a0032916

DeWolf, Melissa, \& Vosniadou, Stella. (2011). The whole number bias in fraction magnitude comparisons with adults. Paper presented at the Cognitive Science Society.

DeWolf, Melissa, \& Vosniadou, Stella. (2015). The representation of fraction magnitudes and the whole number bias reconsidered. Learning and Instruction, 37, 39-49. doi:10.1016/j.learninstruc.2014.07.002

Dunbar, Kevin, Fugelsang, Jonathan, \& Stein, Courtney. (2007). Do naïve theories ever go away? Using brain and behavior to understand changes in concepts. In M. C. Lovett \& P. Shah (Eds.), Carnegie mellon symposia on cognition. Thinking with data (pp. 193-205). Mahwah, NJ, US: Lawrence Erlbaum Associates Publishers.

Durkin, Kelley, \& Rittle-Johnson, Bethany. (2015). Diagnosing misconceptions: Revealing changing decimal fraction knowledge. Learning and Instruction, 37, 21-29. doi:10.1016/j.learninstruc.2014.08.003

Elia, Iliada, Gagatsis, Athanasios, \& Demetriou, Andreas. (2007). The effects of different modes of representation on the solution of one-step additive problems. Learning and Instruction, 17(6), 658-672. doi:10.1016/j.learninstruc.2007.09.011

Espy, Kimberly Andrews, McDiarmid, Melanie M., Cwik, Mary F., Stalets, Melissa Meade, Hamby, Arlena, \& Senn, Theresa E. (2004). The contribution of executive functions to emergent 
mathematic skills in preschool children. Developmental Neuropsychology, 26(1), 465-486. doi:10.1207/s15326942dn2601_6

Field, Andy. (2013). Discovering statistics using ibm spss statistics. London: Sage Publications Ltd.

Gilmore, Camilla, Keeble, Sarah, Richardson, Sophie, \& Cragg, Lucy. (2015). The role of cognitive inhibition in different components of arithmetic. ZDM: The International Journal on Mathematics Education, 47(5), 771-782. doi:10.1007/s11858-014-0659-y

Gómez, David Maximiliano, Jiménez, Abelino, Bobadilla, Roberto, Reyes, Cristián, \& Dartnell, Pablo. (2015). The effect of inhibitory control on general mathematics achievement and fraction comparison in middle school children. ZDM: The International Journal on Mathematics Education, 47(5), 801-811. doi:10.1007/s11858-015-0685-4

Hackman, D. A., Gallop, R., Evans, G. W., \& Farah, M. J. (2015). Socioeconomic status and executive function: Developmental trajectories and mediation. Developmental Science, 18(5), 686-702. doi:10.1111/desc.12246

Kallai, Arava Y., \& Tzelgov, Joseph. (2011). When meaningful components interrupt the processing of the whole: The case of fractions. Acta Psychologica, 139(2). doi:10.1016/j.actpsy.2011.11.009

Lai, Mun Yee, \& Wong, Jeffrey P. (2017). Revisiting decimal misconceptions from a new perspective: The significance of whole number bias in the chinese culture. Journal of Mathematical Behavior, 47, 96-108. doi:10.1016/j.jmathb.2017.07.006

Lortie-Forgues, Hugues, Tian, Jing, \& Siegler, Robert S. (2015). Why is learning fraction and decimal arithmetic so difficult? Developmental Review, 38, 201-221. doi:10.1016/j.dr.2015.07.008

MacLeod, Colin M. (2007). The concept of inhibition in cognition: American Psychological Association.

Mareschal, Denis. (2016). The neuroscience of conceptual learning in science and mathematics. Current Opinion in Behavioral Sciences, 10, 114-118.

Masson, Steve, Potvin, Patrice, Riopel, Martin, \& Foisy, Lorie-Marlène Brault. (2014). Differences in brain activation between novices and experts in science during a task involving a common misconception in electricity. Mind, Brain, and Education, 8(1), 44-55. doi:10.1111/mbe.12043

Mayer, Richard E., Heiser, Julie, \& Lonn, Steve. (2 001). Cognitive constraints on multimedia learning: When presenting more material results in less understanding. Journal of Educational Psychology, 93(1), 187-198. doi:10.1037/0022-0663.93.1.187

McNeil, Nicole M., \& Alibali, Martha W. (2005). Why won't you change your mind? Knowledge of operational patterns hinders learning and performance on equations. Child Dev, 76(4), 883899. doi:10.1111/j.1467-8624.2005.00884.x

Meert, Gaelle, Gregoire, Jacques, \& Noel, Marie-Pascale. (2010). Comparing the magnitude of two fractions with common components: Which representations are used by 10 - and 12-yearolds? Journal of Experimental Child Psychology, 107(3), 244-259. doi:10.1016/j.jecp.2010.04.008

Merkley, Rebecca, Thompson, Jodie, \& Scerif, Gaia. (2015). Of huge mice and tiny elephants: Exploring the relationship between inhibitory processes and preschool math skills. Frontiers in Psychology, 6, 1903. doi:10.3389/fpsyg.2015.01903

Miyake, Akira, Friedman, Naomi P., Emerson, Michael J., Witzki, Alexander H., Howerter, Amy, \& Wager, Tor D. (2000). The unity and diversity of executive functions and their contributions to complex "frontal lobe" tasks: A latent variable analysis. Cognitive Psychology, 41(1), 49100. doi:10.1006/cogp.1999.0734

$\mathrm{Ni}$, Yujing, \& Zhou, Yong-Di. (2005). Teaching and learning fraction and rational numbers: The origins and implications of whole number bias. Educational Psychologist, 40(1), 27-52. doi:10.1207/s15326985ep4001_3

Ninaus, Manuel, Kiili, Kristian, McMullen, Jake, \& Moeller, Korbinian. (2017). Assessing fraction knowledge by a digital game. Computers in Human Behavior, 70, 197-206. doi:10.1016/j.chb.2017.01.004 
Noble, K. G., McCandliss, B. D., \& Farah, M. J. (2007). Socioeconomic gradients predict individual differences in neurocognitive abilities. Developmental Science, 10(4), 464-480. doi:10.1111/j.1467-7687.2007.00600.x

Nunes, Terezinha, \& Bryant, Peter. (2008). Rational numbers and intensive quantities: Challenges and insights to pupils' implicit knowledge. Anales de Psicología, 24(2), 262.

Obersteiner, Andreas, Van Dooren, Wim, Van Hoof, Jo, \& Verschaffel, Lieven. (2013). The natural number bias and magnitude representation in fraction comparison by expert mathematicians. Learning and Instruction, 28, 64-72. doi:10.1016/j.learninstruc.2013.05.003

Resnick, Lauren B., Nesher, Pearla, Leonard, Francois, Magone, Maria, Omanson, Susan, \& Peled, Irit. (1989). Conceptual bases of arithmetic errors: The case of decimal fractions. Journal for Research in Mathematics Education, 20(1), 8-27. doi:10.2307/749095

Roell, M., Viarouge, A., Houde, O., \& Borst, G. (2017). Inhibitory control and decimal number comparison in school-aged children. PLoS One, 12(11), e0188276. doi:10.1371/journal.pone.0188276

Siegler, R. S., Duncan, G. J., Davis-Kean, P. E., Duckworth, K., Claessens, A., Engel, M., Susperreguy, M. I., \& Chen, M. (2012). Early predictors of high school mathematics achievement. Psychol Sci, 23(7), 691-697. doi:10.1177/0956797612440101

Spielberg, Jeffrey M., Galarce, Ezequiel M., Ladouceur, Cecile D., McMakin, Dana L., Olino, Thomas M., Forbes, Erika E., Silk, Jennifer S., Ryan, Neal D., \& Dahl, Ronald E. (2015). Adolescent development of inhibition as a function of ses \& gender: Converging evidence from behavior \& fmri. Human brain mapping, 36(8), 3194-3203. doi:10.1002/hbm.22838

Stafylidou, Stamatia, \& Vosniadou, Stella. (2004). The development of students' understanding of the numerical value of fractions. Learning and Instruction, 14(5), 503-518. doi:10.1016/j.learninstruc.2004.06.015

Sweller, John, \& Chandler, Paul. (1994). Why some material is difficult to learn. Cognition and Instruction, 12(3), 185-233.

Vamvakoussi, Xenia, Van Dooren, Wim, \& Verschaffel, Lieven. (2012). Naturally biased? In search for reaction time evidence for a natural number bias in adults. Journal of Mathematical Behavior, 31(3), 344-355. doi:10.1016/j.jmathb.2012.02.001

Vamvakoussi, Xenia, \& Vosniadou, Stella. (2010). How many "decimals" are there between two "fractions"? Aspects of secondary school students' understanding of rational numbers and their notation. Cognition and Instruction, 28(2), 181-209. doi:10.1080/07370001003676603

Vosniadou, Stella. (2014). Examining cognitive development from a conceptual change point of view: The framework theory approach. European Journal of Developmental Psychology, 11(6), 645661. doi:10.1080/17405629.2014.921153

Vosniadou, Stella, loannides, Christos, Dimitrakopoulou, Aggeliki, \& Papademetriou, Efi. (2001). Designing learning environments to promote conceptual change in science. Learning and Instruction, 11(4), 381-419. doi:10.1016/S0959-4752(00)00038-4

Vosniadou, Stella, \& Verschaffel, Lieven. (2004). Extending the conceptual change approach to mathematics learning and teaching. Learning and Instruction, 14(5), 445-451. doi:10.1016/j.learninstruc.2004.06.014 
Table 1. Mean accuracy, RTs and t-tests for the fraction and decimal magnitude comparison trials.

\begin{tabular}{lcccc}
\hline & \multicolumn{2}{c}{ Control } & \multicolumn{2}{c}{ Counterintuitive } \\
\cline { 2 - 5 } & Accuracy (\%) & RT (ms) & Accuracy (\%) & RT (ms) \\
\hline Fractions & $89(.13)$ & $7172(2857)$ & $81(.18)$ & $9165(3324)$ \\
Decimals & $90(.11)$ & $7713(2601)$ & $68(.32)$ & $7980(2766)$
\end{tabular}

Note. Standard deviations in parentheses. 
Table 2. Accuracy and mean RTs for the Stroop and W-A-M tasks

\begin{tabular}{ccc}
\hline Trial type & Accuracy \% & $R T(m s)$ \\
\hline Congruent & $98(2.5)$ & $843(138)$ \\
Incongruent & $95(4.9)$ & $912(136)$ \\
Go & $95(5.3)$ & $208(27)$ \\
No Go & $85(10.8)$ & $\mathrm{n} / \mathrm{a}$ \\
\hline
\end{tabular}

Note. Standard Deviations in parentheses. 
Table 3. Hierarchical regression analyses with fraction and decimal counterintuitive RT across NAL, $L L$ and $H L$ as dependent variable, $N=89$

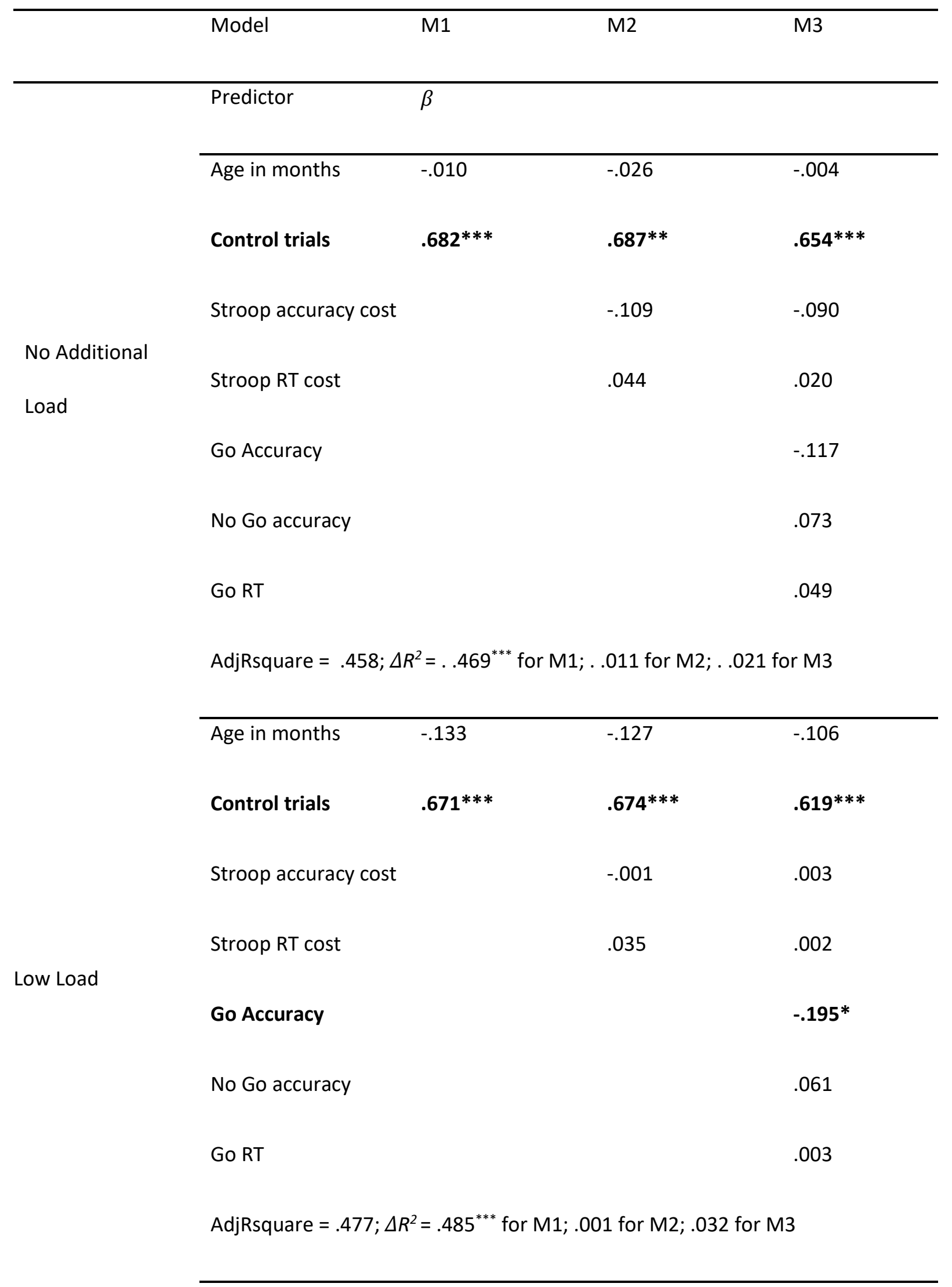




$\begin{array}{llll}\text { Age in months } & -.031 & -.006 & .026 \\ \text { Control trials } & .641^{* * *} & .662^{* * *} & .635^{* * *} \\ \text { Stroop accuracy } & & & \\ \text { cost } & & .184^{*} & .202^{*}\end{array}$

High Load

Stroop RT cost

$-.105$

Go Accuracy

$-.188^{*}$

No Go accuracy

.060

Go RT

.093

AdjRsquare $=.462 ; \Delta R 2=.415^{* * *}$ for $M 1 ; .034$ for M2; .055* for M3

Note. Significant predictors in bold; ${ }^{*} p<.05 .{ }^{* *} p<.01 .{ }^{* * *} p<.001$. 
Figure 1a. Example of No Additional Load trial

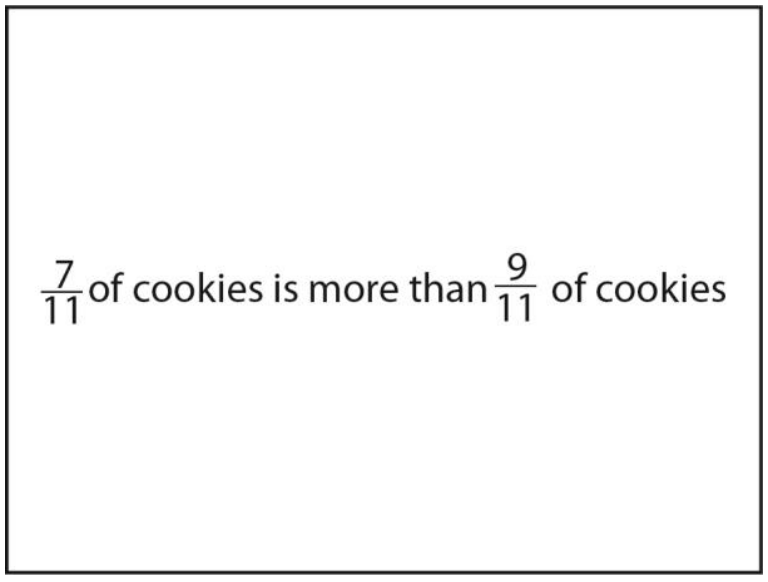

Figure 1b. Example of Low Load trial

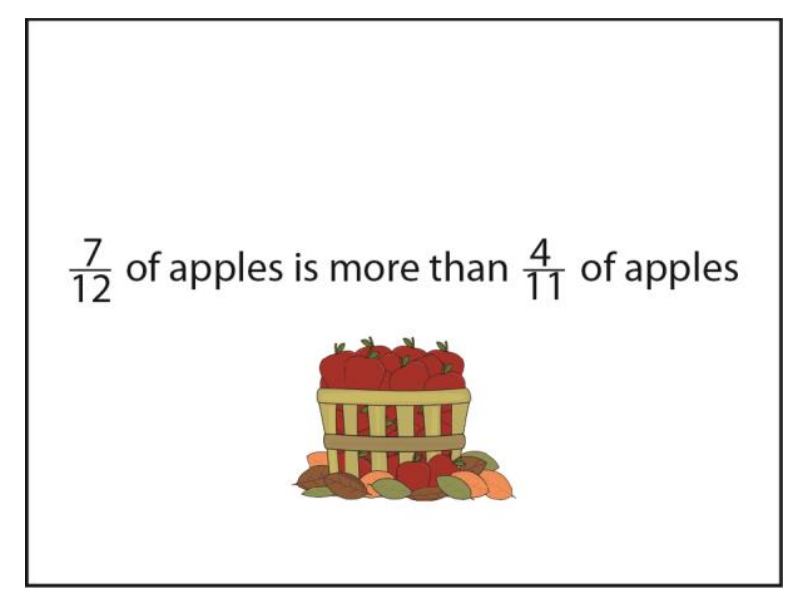

Figure 1c. Example of High Load trial

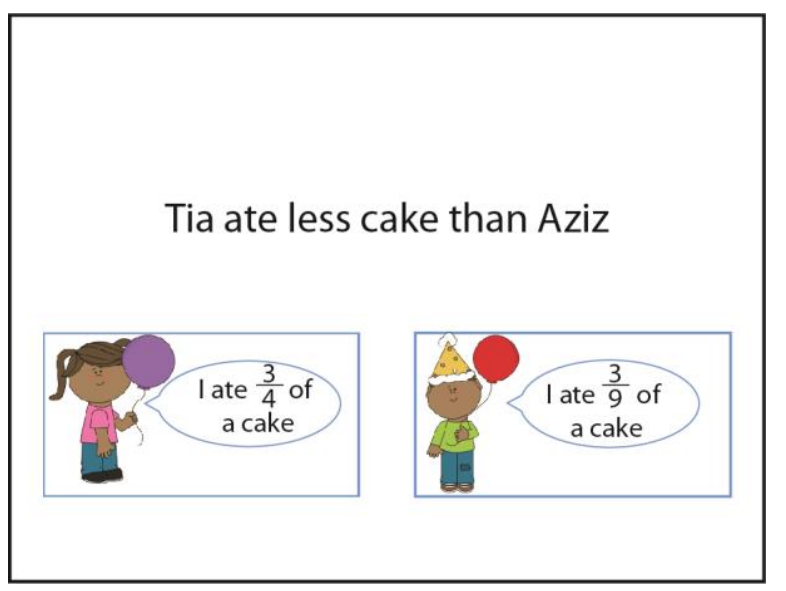


Figure 2. Mean accuracy in Fractions by trial type and cognitive load level. NAL=no additional load, $\mathrm{LL}=$ low load, $\mathrm{HL}=$ high load. Dotted line represents Mean.

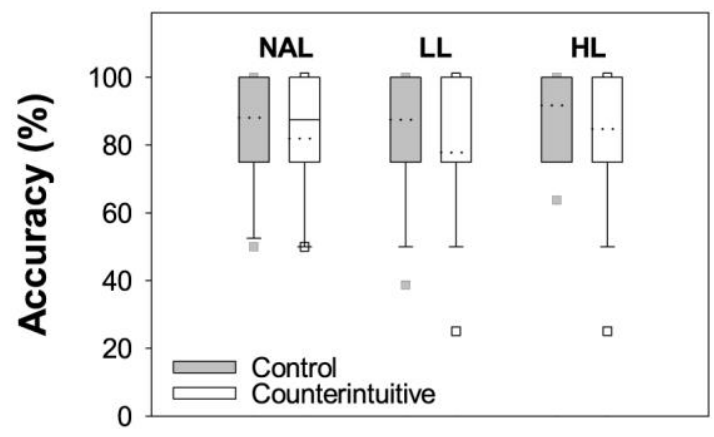

Cognitive Load Condition

Figure 3. Mean Response times (RT) in Fractions by trial type and cognitive load level. Response types are expressed in seconds. NAL=no additional load, $\mathrm{LL}=$ low load, $\mathrm{HL}=$ high load. Dotted line represents Mean.

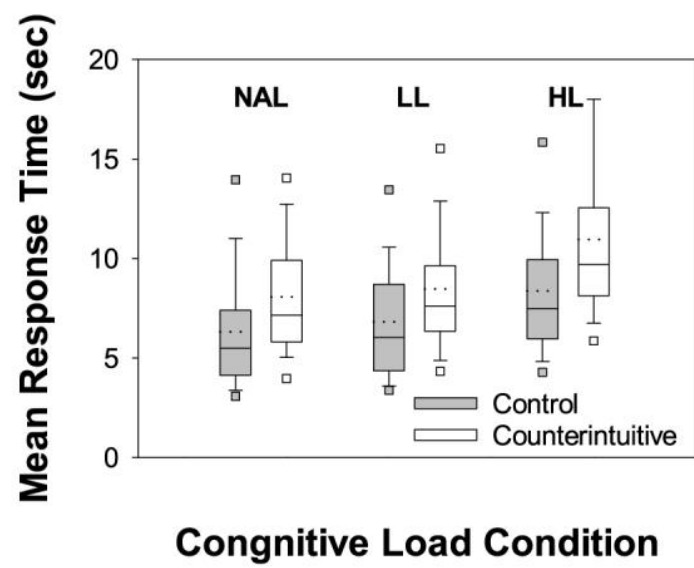


Figure 4. Mean accuracy in decimals by trial type and cognitive load level. NAL=no additional load, LL = low load, HL = high load. Dotted line represents Mean.

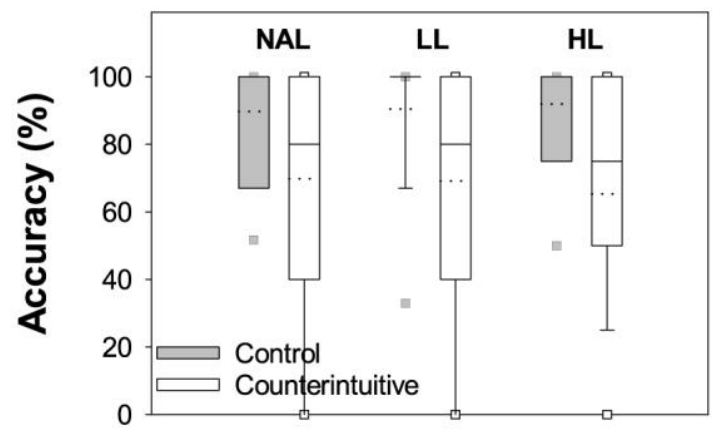

Cognitive Load Condition

Figure 5. Mean Response times (RT) in decimals by trial type and cognitive load level. Response types are expressed in seconds. NAL=no additional load, $\mathrm{LL}=$ low load, $\mathrm{HL}=$ high load. Dotted line represents Mean.

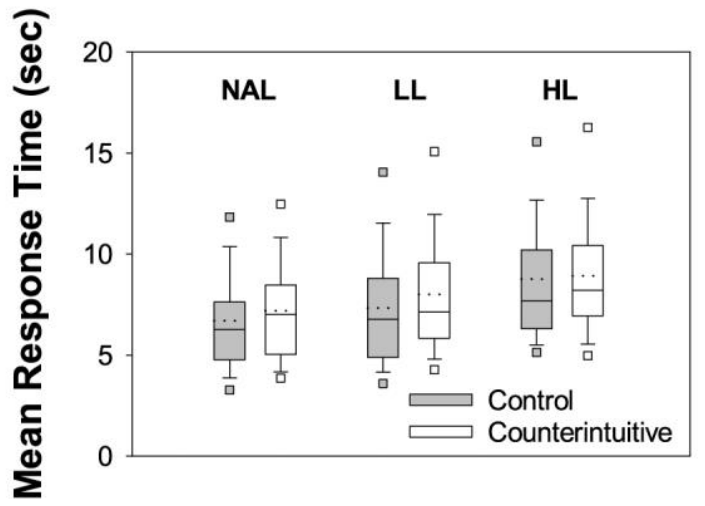

Congnitive Load Condition 